\title{
Disentangling Mathematics from Executive Functions by Investigating Unique Functional Connectivity Patterns Predictive of Mathematics Ability
}

\author{
Kenny Skagerlund ${ }^{1,2}$, Taylor Bolt ${ }^{2}$, Jason S. Nomi ${ }^{2}$, Mikael Skagenholt ${ }^{1}$, \\ Daniel Västfjäll ${ }^{1,3}$, Ulf Träff ${ }^{1}$, and Lucina $Q$. Uddin ${ }^{2}$
}

\begin{abstract}
What are the underlying neurocognitive mechanisms that give rise to mathematical competence? This study investigated the relationship between tests of mathematical ability completed outside the scanner and resting-state functional connectivity (FC) of cytoarchitectonically defined subdivisions of the parietal cortex in adults. These parietal areas are also involved in executive functions (EFs). Therefore, it remains unclear whether there are unique networks for mathematical processing. We investigate the neural networks for mathematical cognition and three measures of EF using resting-state fMRI data collected from 51 healthy adults. Using 10 ROIs in seed to whole-brain voxel-wise analyses, the results showed that arithmetical ability was correlated with FC between the right anterior intraparietal sulcus (hIP1) and the left supramarginal gyrus and between the right posterior intraparietal sulcus (hIP3) and the left middle
\end{abstract}

\section{INTRODUCTION}

Acquiring mathematical competency is one of the most important milestones for individuals in contemporary society. This ability provides the basis for many daily activities, such as shopping, managing a household budget, interpreting cooking recipes, and so on. Still, far from everyone is functionally numerate, and it is estimated that roughly $25 \%$ of the British population suffers from low numeracy (Bynner \& Parsons, 2006). In turn, low numeracy in the population constitutes a major socioeconomical cost to nations (Butterworth, 2010). In the United States population, a large nationwide survey indicates that roughly half of the adult population lack the minimal numerical skills required to use numbers in printed materials, such as calculating change in price menus (Peters, 2012). Therefore, it is essential to investigate how we can foster a fertile learning environment in the early school years and also investigate how these mathematical and cognitive abilities develop into adulthood. Although researchers have made significant progress in trying to understand how mathematical abili-

\footnotetext{
${ }^{1}$ Linköping University, ${ }^{2}$ University of Miami, ${ }^{3}$ Decision Research, Eugene, OR
}

frontal gyrus and the right premotor cortex. The connection between the posterior portion of the left angular gyrus and the left inferior frontal gyrus was also correlated with mathematical ability. Covariates of EF eliminated connectivity patterns with nodes in inferior frontal gyrus, angular gyrus, and middle frontal gyrus, suggesting neural overlap. Controlling for EF, we found unique connections correlated with mathematical ability between the right hIP1 and the left supramarginal gyrus and between hIP3 bilaterally to premotor cortex bilaterally. This is partly in line with the "mapping hypothesis" of numerical cognition in which the right intraparietal sulcus subserves nonsymbolic number processing and connects to the left parietal cortex, responsible for calculation procedures. We show that FC within this circuitry is a significant predictor of math ability in adulthood. ties develop throughout ontogeny in terms of the cognitive abilities (e.g., Mazzocco, Feigenson, \& Halberda, 2011) and neurocognitive substrates (e.g., Price, Yeo, Wilkey, \& Cutting, 2018; Jolles, Ashkenazi, et al., 2016; Park, Li, \& Brannon, 2014) that underlie typical and atypical achievement, surprisingly little is known about the manifestation of adult mathematical competency. In the current project, we formulate the question: What are the underlying neurocognitive correlates that give rise to mathematical competence in adults?

Mathematical calculations have been suggested to rely on a set of core foundational number processing capacities. It is generally acknowledged that humans are equipped with an innate ability to perceive, represent, and manipulate numerosities - the so-called "number sense" (Dehaene, 2011). This preverbal ability has been suggested to constitute the foundation for the symbolic number system (i.e., Arabic digits) used for learning formal arithmetic (e.g., Dehaene, 2011). It is believed there is a mapping of the counting words and visual symbols onto the innate number system as soon as children acquire symbolic language (Dehaene, 2011; Feigenson, Dehaene, \& Spelke, 2004; Xu \& Spelke, 2000). 
Neuroimaging studies have consistently pointed to the intraparietal sulcus (IPS) of the parietal cortex as a focal area implicated in representing abstract meaning of numerosity (e.g., Rosenberg-Lee et al., 2015; Dastjerdi, Ozker, Foster, Rangarajan, \& Parvizi, 2013; Kaufmann, Wood, Rubinsten, \& Henik, 2011; Nieder \& Dehaene, 2009; Ansari, 2008). A study by Harvey, Klein, Petridou, and Dumoulin (2013) using high field fMRI found a topographic representation of numerosity in the right IPS, where neural populations were sensitive to a preferred numerosity and tuning width. Consistent with this, Nieder and Miller (2004) demonstrated that single neurons in the parietal cortex of macaques are tuned to specific numerosities (Nieder \& Miller, 2004). This topographical representation of numerosity gives credence to the notion that humans share with other primates an evolutionary ancient number sense much like any other sensory system (Harvey et al., 2013).

The central role of the IPS was further substantiated by Dastjerdi et al. (2013), who studied three patients with epilepsy. These patients had intracranial implants surgically implanted in the parietal cortex as part of their clinical treatment. Electrocortigography was then used to record the intracranial recordings of neural signaling while administering a set of behavioral tasks. They found numerosity-specific activation in the IPS during an arithmetical task and even during casual social conversations where words signifying quantities were uttered (Dastjerdi et al., 2013). In terms of lateralization, some studies have suggested that the right IPS is predominantly involved in the processing of nonsymbolic quantities whereas the left IPS becomes more involved in processing symbolic quantities throughout development (Holloway, Price, \& Ansari, 2010; Ansari, 2008).

In addition to the involvement of the bilateral IPS, the left angular gyrus (AG) is involved in processing of symbolic numbers and arithmetic facts (Grabner, Reishofer, Koschutnig, \& Ebner, 2011; Price \& Ansari, 2011; Holloway et al., 2010; Ansari, 2008). Price and Ansari (2011) found that passive viewing of Arabic digits elicited activation in the left AG. Teasing out the mechanisms between arithmetic procedures and arithmetic fact retrieval, Grabner et al. (2009) found that the left AG was involved during retrieval of known arithmetic facts while arithmetical procedures elicited a distributed frontoparietal network. In addition, Grabner et al. (2011) found stronger left AG activity in more versus less math competent adults during a task requiring arithmetical calculations, which the authors suggested may reflect their higher proficiency in processing mathematical symbols. The left AG is part of a language network that subserves overlapping processes such as phonological processing and processing of arithmetic facts, and different subregions of the AG may be preferentially involved depending on domain (Andin, Fransson, Rönnberg, \& Rudner, 2015). For example, the posterior AG was more involved during arithmetical procedures, whereas the anterior AG was preferentially involved in language processing (Andin et al., 2015). However, others have found that AG showed increased deactivation during arithmetic and other effortful cognitive tasks (Wu et al., 2009). Along these lines, the AG is part of the default mode network, which is typically deactivated when a salient event detected by the salience network switches to engage the central executive network (Menon \& Uddin, 2010). Thus, some have argued that the engagement of the left AG is related to difficultyrelated modulations of the default mode network, rather than to numerical and arithmetical tasks per se (e.g., Wu et al., 2009). Others have dismissed the domainspecific role of the $A G$ in math and tried to reconcile these different findings into a coherent picture where the AG is believed to be part of a bottom-up attentionto-memory irrespective of domain (cf. Bloechle et al., 2016). Therefore, the role of the AG is still unresolved, although its importance in mathematical cognition is uncontested. One reason for this may be that the anatomical demarcation of the AG, as well as the IPS, in the parietal cortex is difficult. Despite this, a majority of neuroimaging studies regard the IPS and AG as unitary cortical regions. However, cytoarchitectonic mapping studies suggest the IPS can be divided into hIP1, hIP2, and hIP3, and AG can be divided into anterior (PGa) and posterior (PGp) portions (Caspers et al., 2006) and that these subdivisions show distinct patterns of functional and structural connectivity (Uddin et al., 2010). Existing literature that has investigated the specific role of each subdivision of the IPS and AG in mathematics and numerical cognition are scarce (but see Price et al., 2018).

In summary, two regions of the parietal cortex have been reliably tied to basic number processing that in turn provide the foundation for subsequent higher order mathematics, such as arithmetic. These two brain regions have been implicated in number processing and arithmetic across development, although there seems to be a frontoparietal shift in which adults show more posterior activation of the IPS as a function of age (Kaufmann et al., 2011). Still, the functional contribution of each subdivision of the IPS and AG to mathematical cognition is not fully understood.

The bulk of previous research into cognitive development of mathematical skills has primarily focused on activation of isolated brain areas. Recent network neuroscience research indicates that these regions are embedded within functionally specialized networks that interact and reorganize throughout development through consistent coactivation (Menon, 2013). Therefore, it has been increasingly acknowledged that arithmetic-and mathematics more generally-is subserved by a distributed neurocognitive network both within and between multiple functional brains system (Fias, Menon, \& Szücs, 2013). Beyond cortical sites involved in basic number processing, it has been suggested that arithmetic calculations rely on long-term memory systems, working memory processing, and cognitive control (e.g., Arsalidou \& 
Taylor, 2011). In addition, mathematical computations rely on a dorsal attention stream comprising the superior parietal lobe (SPL), motor areas, and middle frontal gyrus (MFG; Supekar \& Menon, 2012; Corbetta \& Shulman, 2002). An informative way of investigating the underlying neural networks subserving mathematical processing is through functional connectivity (FC) analyses. Price et al. (2018) used resting-state fMRI to investigate FC patterns that were predictive of arithmetic ability in children and found that the degree of connectivity between the left and right IPS was predictive of ability, which is consistent FC analyses using task-related fMRI (Park, Park, \& Polk, 2013). The interhemispheric parietal connectivity was taken to suggest that there was a mapping between nonsymbolic numerosities and symbolic representations. Interestingly, the authors also found that the connectivity strength between AG and the frontal pole was negatively related to arithmetic competence, which indicates that frontoparietal connectivity strength is not necessarily advantageous for cognitive processing but may reflect less functional specialization of neural circuits. In fact, recent research of FC patterns in children with mathematical learning disabilities or developmental dyscalculia (MLD/ DD) indicate that the IPS may be hyperconnected to frontal areas, providing a potential biomarker of MLD/DD (Jolles, Ashkenazi, et al., 2016; Rosenberg-Lee et al., 2015).

The areas of the parietal cortex mentioned above are not uniquely involved in mathematics but are also involved in executive functions (EFs), such as inhibitory control, shifting, and working memory processes. This central executive network comprises nodes in the dorsolateral pFC (DLPFC) and the posterior parietal cortex including the IPS (Sherman et al., 2014; Menon \& Uddin, 2010). Previous research has shown that the dorsal attention stream comprising the SPL, motor areas, and the MFG is involved in mathematical computations (Menon \& Uddin, 2010; Corbetta \& Shulman, 2002). Thus, it remains unclear whether there are unique subnetworks for mathematical processing or whether they are completely shared by neural circuits involved in EF. The current study aims to investigate the resting-state FC patterns of cytoarchitectonically defined subdivisions of the parietal cortex and relationships with arithmetical ability in adults while disentangling the contributions of EF ability. Given that the IPS and AG, as well as their respective subdivisions, are foundational for mathematics and number processing, the focus will be on analyzing arithmetic as the principal outcome variable. Measures of $\mathrm{EF}$ will here be used as covariates to investigate unique FC patterns that are predictive of arithmetical ability.

\section{METHODS}

\section{Participants}

Fifty-one healthy adult participants ( $n=51,28$ women and 23 men, mean age $=23.43$ years, $S D=2.82$ ) participated in the fMRI study and were recruited and enrolled at Linköping University. The participants were all students, where $63 \%$ of the students were from the faculty of humanities and social sciences and $37 \%$ of the students were from the faculty of engineering and natural sciences. All participants had normal or corrected-tonormal vision and reported no history of drug abuse or neurological illness. The participants were paid a sum corresponding to $\$ 60$ for their participation. All participants provided written and informed consent before scanning. The study was approved by the regional ethics committee of Region Östergötland, Sweden. See Table 1 for an overview of demographics and descriptive statistics of the tasks used in the study.

\section{Cognitive Measures}

The behavioral tests were all administered on a separate day before the scanning session. All participants completed the tests in the same order: (1) inhibition task, (2) arithmetic calculation task, (3) working memory task, and (4) switching task. The measures are described in full below.

\section{Mathematics-Arithmetic}

Arithmetic calculation ability was assessed using a similar procedure as Gebuis and van der Smagt (2011) and

Table 1. Descriptive Statistics and Correlations among the Measures

\begin{tabular}{|c|c|c|c|c|c|c|}
\hline \multirow[b]{2}{*}{ Tasks } & \multirow[b]{2}{*}{$M$} & \multirow[b]{2}{*}{$S D$} & \multicolumn{4}{|c|}{ Correlation Coefficients } \\
\hline & & & 2 & 3 & 4 & 5 \\
\hline 1. Age (in years) & 23.43 & 2.84 & -.04 & .03 & -.06 & -.13 \\
\hline 2. Arithmetic (raw score out of 189) & $103.67(55 \%)$ & 22.82 & - & -.28 & -.46 & .37 \\
\hline 3. $\mathrm{EF}$-inhibition (sec) & 25.50 & 4.44 & & - & .36 & .15 \\
\hline 4. $\mathrm{EF}$ —shifting (sec) & 40.23 & 14.01 & & & - & -.32 \\
\hline 5. WAIS—digit span (raw score) & 27.37 & 4.96 & & & & - \\
\hline
\end{tabular}

Significant correlations in bold $(p<.05)$. 
Lindskog, Winman, and Poom (2017). This test was divided into four subtests (addition, subtraction, multiplication, and division). For each subtest, the participants were presented with a sheet of paper containing printed arithmetic problems of increasing difficulty. For each subtest, they were instructed to complete as many problems as they could within the allotted time of 120 sec. A brief pause was included in between each subtest. The difficulty level of the problems was manipulated by increasing the number of digits or by requiring borrowing or carrying. Each subtest contained 54 problems, except for the division task that contained 27 problems. In summary, the maximum score was 189 . The total number of correctly solved arithmetic problems across all four conditions was used as a measure of arithmetic calculation ability.

\section{EFs-Inbibition}

Inhibition was measured using a traditional color Stroop task. Each participant was presented with a piece of paper with 30 color words written in two separate columns. This test was divided into two conditions: one with congruent color words and one with incongruent color words. Each condition was completed twice, resulting in 60 words in each of the two conditions. The participant was to identify and read out loud as fast as possible the color with which the words were written. Immediately upon completion of one round of 30 words of a given condition, the participant went on to the next round when ready. An experimenter used a stopwatch to measure the time in seconds it took to read all 30 words in each round in each condition. The mean response time of the incongruent condition was used as an index of inhibition.

\section{EFs-Shifting}

Shifting ability was assessed using a paper-and-pencil version of the Trail Making Test (van der Sluis, de Jong, \& van der Leij, 2004), which contained two conditions. The first condition (A) contained 22 circles, each containing a digit, whereas the second condition (B) also contained 22 circles but now with either a digit or a letter written in it. In Condition A, the task was to draw a line and connect the circles in ascending order as fast as possible. In Condition $\mathrm{B}$, the participants are told to draw the line and connect the circles in ascending order once again, but now in alternating order (1-A-2-B-3-C, etc.) and as quickly as possible without making any mistakes. The dependent measure was the number of seconds for each condition it took for the participant to connect all of the circles. The condition requiring shifting between digits and letters was used as index of shifting ability.

\section{EFs_Working Memory}

The working memory subcomponent of EF was assessed using the digit span subtest of Wechsler Adult Intelligence
Scale-IV (WAIS-IV; Wechsler, 2008). This subtest is divided into three conditions: digit span forward, digit span backward, and digit span sequencing. In the first condition, the participant hears a series of digits and attempts to repeat them out loud in order. In contrast, in the digit span backward condition, the participant has to repeat the string of digits in reverse order. The sequencing condition requires the participant to recall all the digits in correct ordinal sequence. All conditions become increasingly more difficult in terms of the number of digits there are to be repeated. The maximum score for each condition is 16 , for a total of 48 for the entire task.

\section{fMRI Data Acquisition}

The fMRI data were collected at the Center for Medical Imaging and Visualization at Linköping University in Sweden. Images were acquired using a 3.0-T Siemens Magnetom Prisma MRI scanner fitted with a 20-channel head coil. Forty-eight $3.0 \times 3.0 \times 3.0 \mathrm{~mm}$ thick slices with an in-plane resolution of $3.0 \mathrm{~mm}$ isotropic and no gap were acquired. A BOLD-sensitive T2*-weighted ascending EPI pulse sequence was used to acquire wholebrain functional scans (repetition time $=1340.0 \mathrm{msec}$, echo time $=30.0 \mathrm{msec}$, flip $=69^{\circ}$ ). The entire restingstate EPI sequence lasted for $10 \mathrm{~min}$, resulting in a total of 450 volumes acquired per participant. The participants were instructed to keep their eyes open and fixate on a white fixation cross for the duration of the scan. The resting-state sequence was collected as part of a larger data collection involving task-related data not reported here. The participants also performed a numerical comparison task after which a risk perception task was performed. These tasks were administered before a brief rest followed by the 10-min resting-state scan. A diffusion tensor imaging sequence concluded the data collection, which is also not reported here.

\section{fMRI Preprocessing}

The resting-state fMRI data were preprocessed using the Data Processing and Analysis of Brain Imaging toolbox (DPABI; Yan, Wang, Zuo, \& Zang, 2016) (rmfri.org/ DPABI), which is based on SPM (www.fil.ion.ucl.ac.uk/ spm). The first five volumes were discarded to allow for T1 equilibration. Temporal and head motion correction was applied on the remaining volumes, which were then warped into standard Montreal Neurological Institute (MNI) space at the resolution of $3 \times 3 \times 3 \mathrm{~mm}$. FC analyses are particularly sensitive to spurious results due to head motion. To mitigate these effects, we followed recommendations by Power and colleagues to compute framewise displacement (FD; Power et al., 2014; Power, Barnes, Snyder, Schlaggar, \& Petersen, 2012). The average FD metric was used to further reduce the effects of head motion artifacts by scrubbing the data. We used the cut option in the DPARSF toolbox according to the 
following parameters: FD $=0.5$ as the threshold for bad time points, scrubbing time points before bad time points $=1$, scrubbing time points after bad time points $=2$ (Power et al., 2012). This procedure identified a mean FD of $>0.5$ in $1.9 \%$ in of our current sample. These bad time points were subsequently scrubbed according to the aforementioned parameters. Participants had an average of $410.96(S D=54.85)$ volumes remaining after scrubbing. All normalized images were smoothed with a Gaussian kernel (FWHM $6 \mathrm{~mm}$ ) and then detrended. Signals from white matter, cerebrospinal fluid, and 24 rigid body motion parameters were regressed out of the data (Friston, Williams, Howard, Frackowiak, \& Turner, 1996). A bandpass filter $(0.01-0.1 \mathrm{~Hz})$ was then applied to the regressed time series.

\section{fMRI Data Analysis}

\section{FC Analysis}

ROI selection For both hemispheres, we defined the seeds in IPS (hIP1, hIP2, and hIP3) and AG (PGa and PGp) using the cytoarchitectonic probability maps of the Anatomy Toolbox in SPM12. For information about anatomical boundaries of these maps, see Caspers et al. (2008) and Choi et al. (2006). See Figure 1 for visual representations of these ROIs. These 10 ROIs were used as seeds in the resting-state FC analyses.
Subject level ROI-to-whole-brain connectivity analyses. For each participant, we extracted the mean time-series of each ROI using the FC voxel-wise option in the REST toolbox (www.restfmri.net/forum/REST). A whole-brain FC map was created for each participant for each seed region by computing the bivariate correlation coefficient between the mean time course from a given seed region and the time course of every other voxel in the brain. These FC maps were then transformed using Fisher's $r$-to- $z$ transformation, which converts the Pearson's $r$ values to normally distributed $z$ scores using the formula $z^{\prime}=0.5 *[\ln (1+r)-$ $\ln (1-r)]$.

Initial contrasts of FC of IPS and AG subdivisions Before investigating the group-level connectivity correlates of arithmetic ability and EFs, we contrasted each subdivision within the IPS and AG separately to examine the correspondence to previous studies in adults (Uddin et al., 2010) and in children (Price et al., 2018). The individual $z$-transformed FC maps were used in a second-level random effects general linear model (GLM). These results are provided in more detail in the supplementary materials $^{1}$ (Table S1 and Figure S1).

Group-level FC correlates of arithmetic ability To investigate the unique and overlapping FC patterns related to arithmetic ability, we conducted second-level group
Figure 1. Renditions, axial and coronal slices illustrating the ROIs in the left hemisphere derived from cytoarchitectonic maps for (A) IPS: hIP1 (red), hIP2 (blue), and hIP3 (green). ROIs derived from cytoarchitectonic maps for (B) AG: PGa (violet) and PGp (yellow).

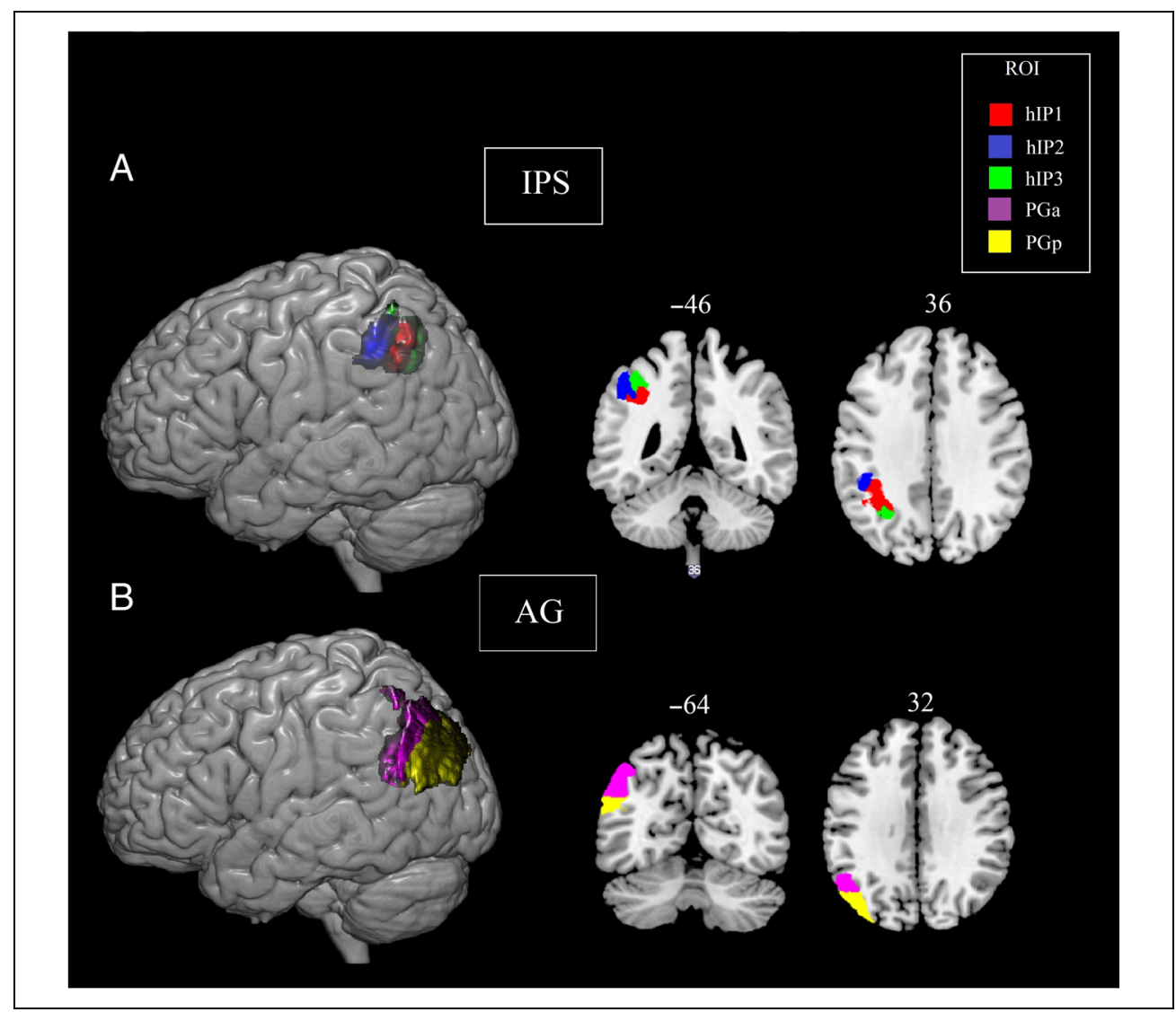


analyses in two steps. First, we performed regression analyses in FSL by initially inserting only scores of arithmetic as outcome variable in the GLM without any covariates. Scores of arithmetic ability were then correlated with participant-level FC maps for each seed. This analysis reveals overall $\mathrm{FC}$ patterns that are predicted by arithmetic performance, irrespective of whether these connectivity patterns and nodes are reflective of number processing components or EFs. In a second step, we included the three measures of EF as nuisance covariates in the GLM and then correlated participant-level FC maps again with arithmetic ability. This second model was meant to disentangle the role of nodes in a network more strongly related to mathematical processing, while controlling for the potential neurocognitive overlap between EF and arithmetic. Results were masked using a gray matter image and thresholded at $p<.001$ (voxel-wise, uncorrected), with family-wise error (FWE) corrections for multiple comparisons at the cluster level $(p<.05)$.

\section{RESULTS}

\section{FC Contrasts: Intraparietal Sulcus: hIP1, hIP2, hIP3}

The left and right hIP1 showed strong FC with other areas of the inferior parietal lobule. This included both intrahemispheric and intershemispheric connections with the AG, compared with hIP2 and hIP3. The left and right hIP1 showed strong FC to areas of the temporal gyri compared with hIP2 and hIP3. Right hIP1 showed strong connectivity with the left inferior frontal gyrus (IFG) compared with hIP2 and hIP3. The hIP3 displayed stronger FC with occipital areas, the left orbital gyri, precentral gyri, and temporal gyri, compared with hIP2, which instead showed stronger FC to bilateral IFG and to supramarginal gyrus (SMG; see Table S1 and Figure S1 for details).

\section{FC Contrasts: AG: PGa, PGp}

Both left and right PGp showed consistent connectivity with occipital areas and anterior and mid cingulate areas compared with PGa. The right PGp demonstrated stronger correlation with several clusters located in pFC, such as MFG bilaterally and with the left IFG compared with the PGa. The right PGa showed stronger connectivity with the left inferior parietal areas, such as the left AG and clusters extending to the posterior and mid cingulate. The left PGa also showed connectivity with the right caudate nucleus and temporal gyri compared with the PGp (see Table S1 and Figure S1).

\section{FC and Arithmetic Ability}

Investigating the overall correlation between arithmetic ability and our ROIs, our regression analysis showed that all of the associations could be identified in the right IPS and the left AG. The right hIP1 showed significant connectivity with the left SMG positively related to arithmetic, whereas connectivity between the right hIP1 and the right superior frontal gyrus (SFG) was negatively associated with arithmetic. Connectivity of the posterior portion of the right IPS and hIP3 with the left MFG and the right precentral gyrus around the area of premotor cortex (PMC) was significantly correlated with arithmetic. Only one subdivision of the AG showed FC that was associated with arithmetic ability, and that was the left PGp that showed significant connectivity with the left IFG. Of note is the intraparietal and contralateral connectivity pattern between hIP1 and SMG. See Table 2 and Figure 2 for an overview of this analysis.

\section{FC and Arithmetic Ability When Controlling for EF}

To investigate the specificity of the FC patterns between the ROIs and the target clusters to arithmetical ability, we included three measures of EF as covariates in the GLM. While controlling for all these aspects of EF, our regression analysis revealed a new pattern of results compared with the previous analysis above. Like before, however, hIP1 showed significant connectivity with the left SMG that was positively related to arithmetic. This analysis also revealed that the connectivity between the right hIP3 and both left and right PMC was associated with arithmetic, while controlling for EF. The connectivity between the left hIP3 and the left PMC showed significant association with arithmetic ability. This analysis highlights the specificity of intraparietal and contralateral FC between right hIP3 and left SMG for mathematics.

Table 2. Functional Connectivity Patterns between Seed ROIs and Regions that Are Correlated with Arithmetic Ability

\begin{tabular}{llcrrr}
\hline Seed Region & \multicolumn{1}{c}{ Target Region } & MNI & Cluster Size & $p$ & $z$ Score \\
\hline R hIP1 & L SMG & $-55,-33,30$ & 86 & 4.91 \\
R hIP1 & R SFG & $21,42,54$ & 52 & $<.001$ \\
R hIP3 & L MFG (DLPFC) & $-42,42,30$ & 39 & $<.001$ \\
R hIP3 & R precentral gyrus (PMC) & $27,-6,51$ & 107 & $<.71$ \\
L PGp & L IFG & $-45,36,18$ & 44 & $<.001$ \\
\hline
\end{tabular}

$p<.001$ uncorrected at voxel level and cluster level corrected at $p<.05 . \mathrm{R}=$ right; $\mathrm{L}=$ left. 


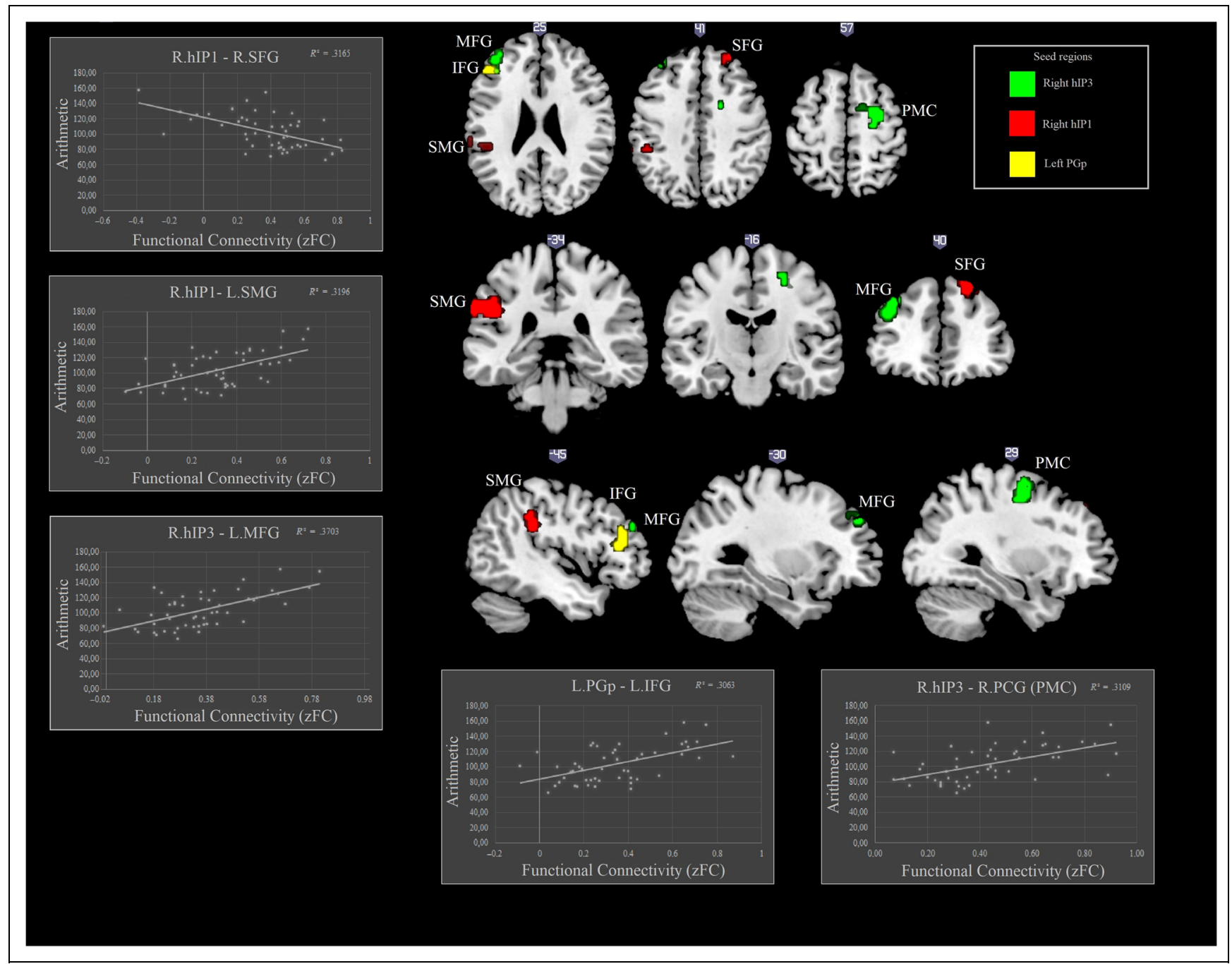

Figure 2. Functional connectivity maps that are statistically significant predictors of arithmetic at $p<.001$ at voxel level (uncorrected) and $p<.05 \mathrm{FWE}$ corrected at the cluster level.

See Table 3 and Figure 3 for an overview of this analysis (Figures 4 and 5).

\section{DISCUSSION}

Unique contributions of parietal-based networks to mathematical cognition and EF are incompletely understood. The purpose of the current study was to contribute to our understanding of intrinsic FC patterns that subserve arithmetical ability above and beyond EF. No previous study has used intrinsic connectivity patterns in adults obtained using resting-state fMRI data to address the issue of disambiguating the associations of mathematical competence and EFs with brain activity. To address this, we analyzed resting-state FC of specific subdivisions of the IPS and the AG, known to be pivotal areas involved in

Table 3. Functional Connectivity Patterns between Seed ROIs and Regions that Are Correlated with Arithmetic Ability When Controlling for EF

\begin{tabular}{|c|c|c|c|c|c|}
\hline Seed Region & Target Region & $M N I$ & Cluster Size & $p$ & $z$ Score \\
\hline R hIP1 & L SMG & $-55,-33,30$ & 53 & $<.001$ & 5.19 \\
\hline L hIP3 & L SFG (PMC) & $-18,6,60$ & 39 & $<.001$ & 4.71 \\
\hline R hIP3 & L SFG (PMC) & $-18,6,60$ & 51 & $<.001$ & 4.32 \\
\hline R hIP3 & $\mathrm{R}$ posterior medial frontal (PMC) & $15,0,51$ & 103 & $<.001$ & 4.49 \\
\hline
\end{tabular}

$p<.001$ uncorrected at voxel level and cluster level corrected at $p<.05 . \mathrm{R}=$ right; $\mathrm{L}=$ left. 
Figure 3. Overview of FC patterns predictive of arithmetical ability. Nodes in red signify positive correlations with arithmetic and blue signify negative correlations

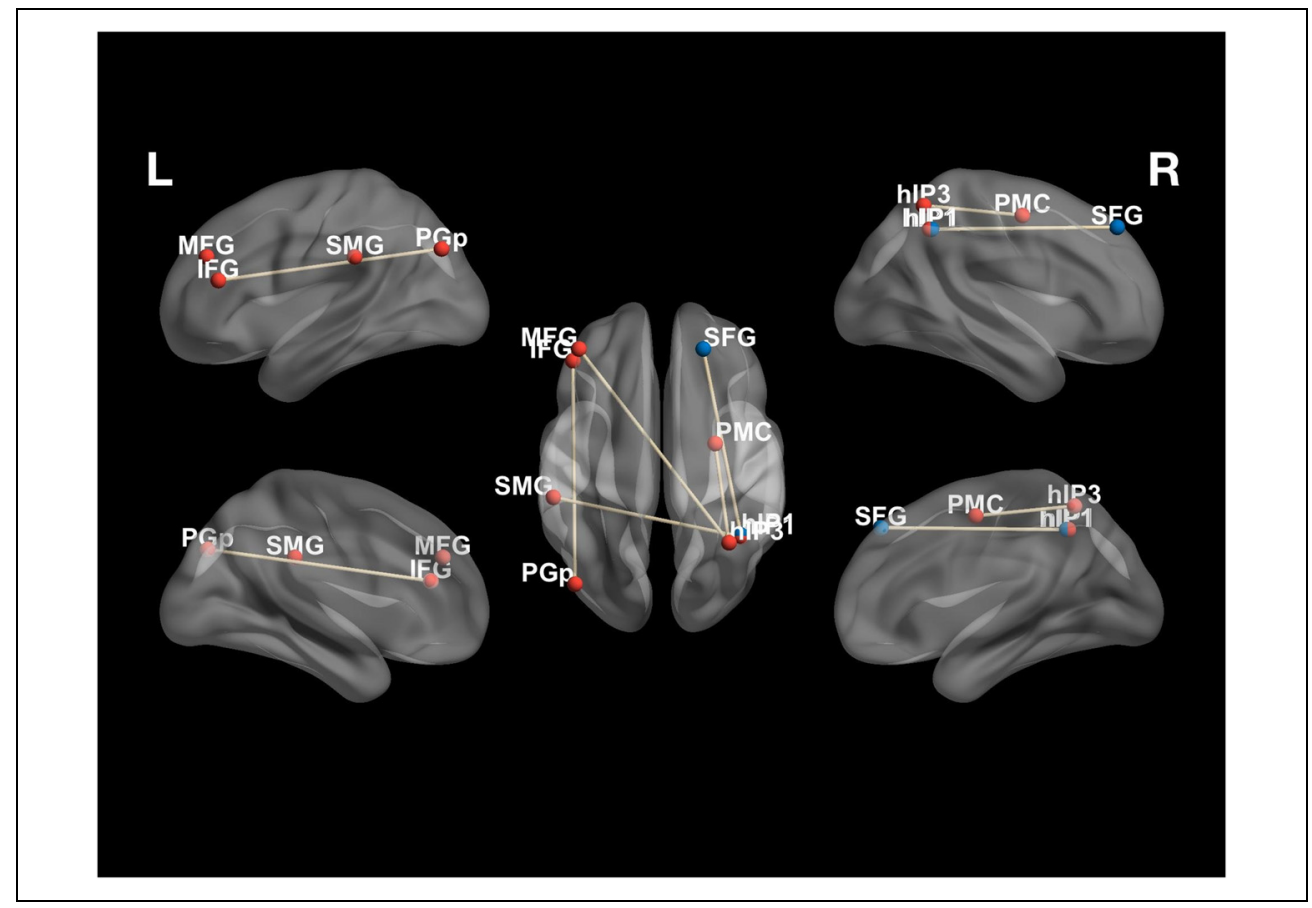

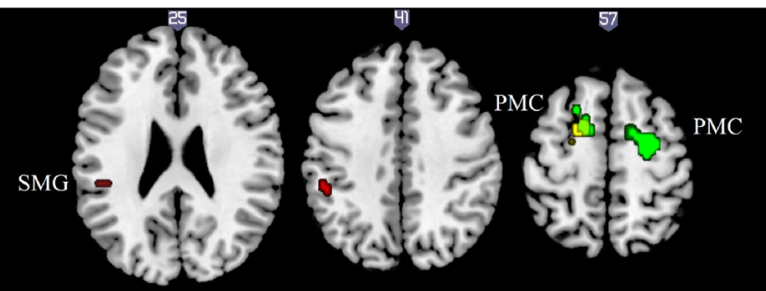

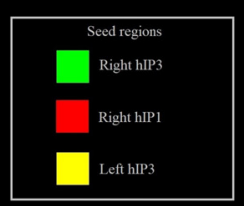

Functional Connectivity (zFC)

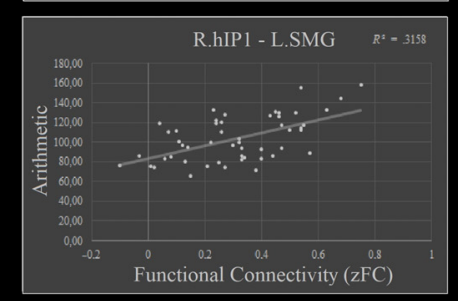

R. hIP3 - L.PCG (PMC) $\quad R^{x}=2373$
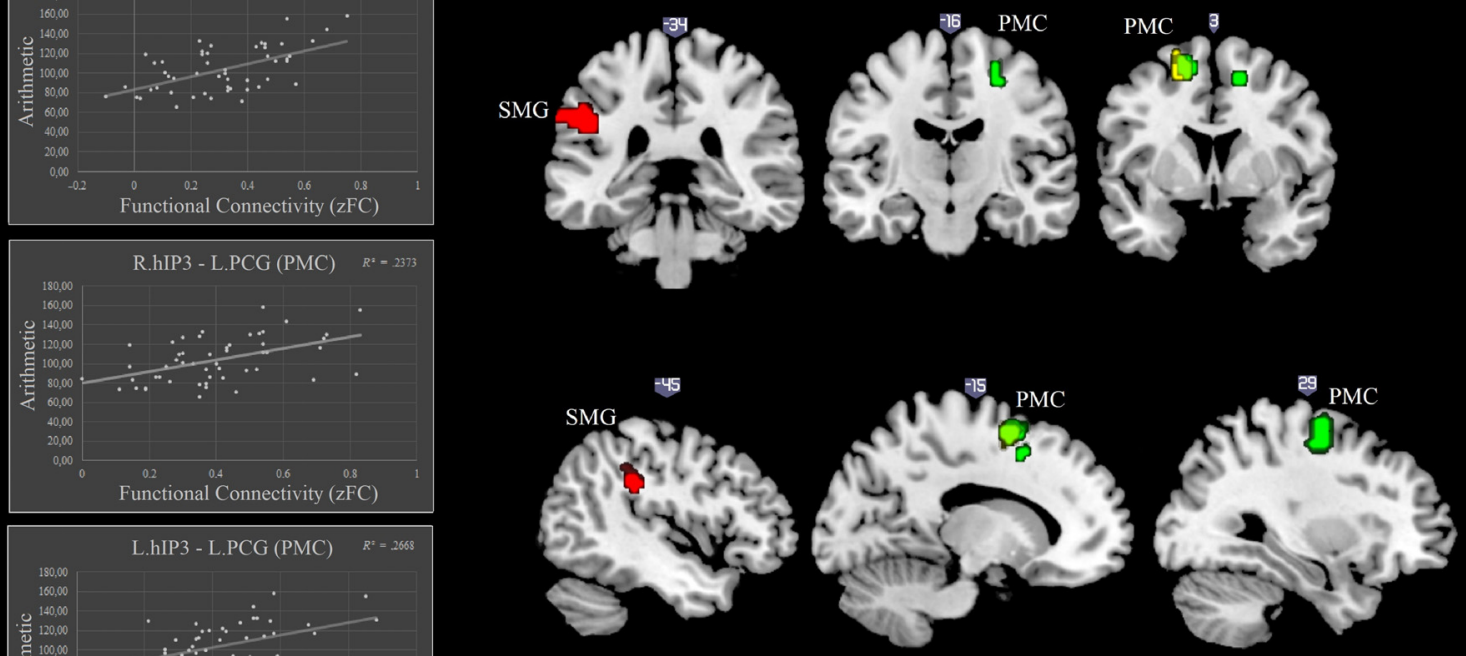

Functional Connectivity $(\mathrm{zFC})$

Figure 4. Functional connectivity maps that are statistically significant predictors of arithmetic at $p<.001$ at the voxel level and $p<.05$ FWE corrected at the cluster level. 
Figure 5. Overview of FC patterns that are positively related to arithmetical ability when controlling for three measures of EFs.

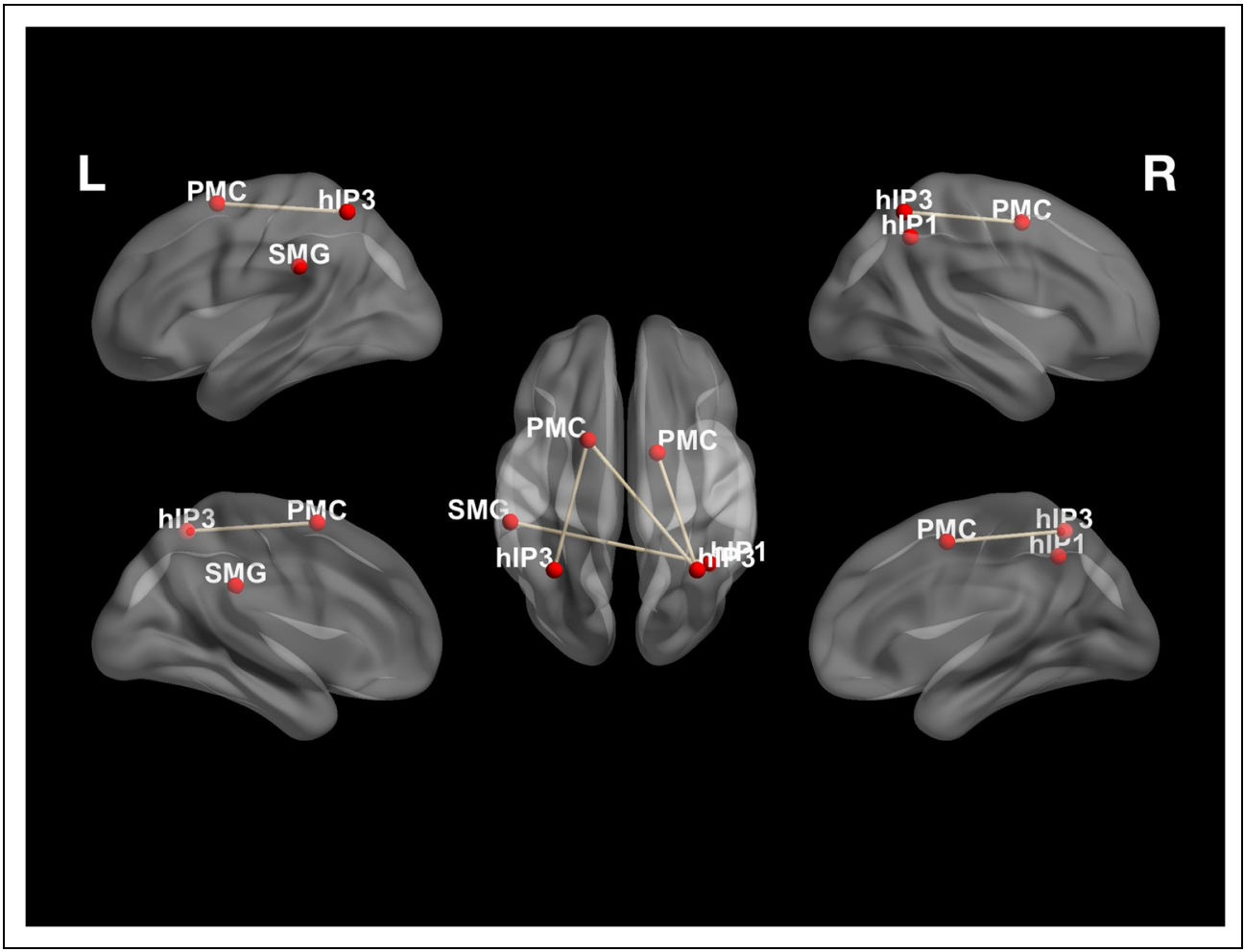

numerical processing, and correlated FC with arithmetical ability in a large sample of adults. These areas of the parietal cortex are also involved in EFs such as inhibitory control, shifting, and working memory processes in the central executive network (Sherman et al., 2014; Menon \& Uddin, 2010). Thus, a central aim of this study was to disentangle intrinsic connectivity patterns that subserve arithmetical ability when accounting for EF. Before the main analyses, we determined the overall FC pattern of each parietal subdivision, allowing for comparison between previous studies (Price et al., 2018; Uddin et al., 2010) Overall, the connectivity pattern of each subdivision was similar to both previous studies. The decision was then made to investigate the connectivity patterns in a two-step process. In the first step of our analyses, we investigated what FC patterns that were predictive of arithmetical ability overall, without including the measures of EF as covariates in the GLM. Thus, investigating intrinsic connectivity patterns without controlling for $\mathrm{EF}$ is a valid means of examining what networks are important for efficient mathematical computations in general. However, it is also of interest to unveil regional activity patterns that are more pronounced in mathematics than in $\mathrm{EF}$, thus giving insight into unique and overlapping connectivity patterns underlying these processes.

\section{FC and Arithmetic Ability}

Our results demonstrate that the strength of FC between the posterior portion of the right IPS and contralateral SMG is significantly related to arithmetic ability. Our results are overall consistent with research on children, where contralateral connectivity patterns are consistently found in the parietal cortex both in intrinsic (Price et al., 2018) and evoked (Park et al., 2013) studies of FC. However, whereas Price and colleagues (2018) found IPS-to-IPS connectivity in children to be associated with arithmetical ability, our adult sample showed no such coupling. This discrepancy could be due to the different ages of the samples. In their task-related fMRI, Park et al. (2013) used psychophysiological interaction analysis in a sample of adults that showed evoked connectivity coupling between the right IPS and the left IPS that extended to the AG. The functional coupling between the left and the right IPS has often been interpreted as reflecting a mapping between non-symbolic magnitudes subserved by the right IPS and a symbolic number system primarily processed by the left IPS or AG. This notion is consistent with the conceptual idea that counting words and visual symbols are believed to map onto the innate number system during childhood (Dehaene, 2011; Xu \& Spelke, 2000; Feigenson et al., 2004). Indeed, one influential hypothesis regarding the characterization of developmental dyscalculia is that this specific learning disorder is characterized by a dysfunctional connection between these two systems (e.g., De Smedt \& Gilmore, 2011). With respect to the developmental trajectory of the neurocognitive sites and networks that underlie number processing, studies indicate that there is a general frontoparietal shift across development. Although children tend to recruit areas of the pFC during number processing, they also seem to recruit more anterior portion 
of the left IPS to a greater extent than adults, who instead show a more posterior activation of the IPS (Kaufmann et al., 2011). Thus, it could be the case that in our sample of adults the FC pattern between the right IPS and the symbols they represent, typically activated by the AG or left IPS, is no longer predictive of arithmetical ability. Instead, the functional coupling between the right IPS and the left SMG may indicate a stronger establishment between magnitudes and the language-driven symbol system. Studies have consistently found the left SMG to be involved during mental computation (Grabner et al., 2007; Ischebeck et al., 2006; Dehaene, Spelke, Pinel, Stanescu, \& Tsivkin, 1999), so one tentative interpretation is that the connectivity pattern between the IPS and the SMG reflects efficient transformation processes from nonsymbolic magnitudes to verbal symbols that can be used for explicit mathematical computations. This would be in line a study by Park et al. (2014), who demonstrated evoked connectivity patterns between the right SPL and the left SMG during symbolic number processing in children. In contrast, in a group of children with math difficulties, Jolles, Ashkenazi, et al. (2016) found that the IPS was hyperconnected with the right SMG and to dorsal prefrontal regions. This is also consistent with a meta-analysis showing greater right SMG engagement in children with learning difficulties during numerical processing (Kaufmann et al., 2011). Thus, there seems to be a differentiation between the connectivity patterns of the IPS and the SMG in terms of lateralization. Whereas stronger FC between the IPS and the left SMG may be indicative of efficient integration of number magnitudes and language processing, stronger FC between the IPS and the right SMG and prefrontal regions may indicate that dorsal and ventral attention systems are less segregated and specialized.

Corroborating findings reported by Park and colleagues (2013), we found that the connectivity between the right IPS and the left sensorimotor region was related to arithmetic. In terms of resting-state FC, connectivity analyses have demonstrated intrinsic connectivity patterns between the IPS, the PMC, and the MFG (Uddin et al., 2010). It has been argued that the consistent activation of the PMC during numerical processing could be due to subvocalization or finger counting (e.g., Piazza, Mechelli, Price, \& Butterworth, 2006). The role of the PMC and FEFs in dorsal attention network is firmly established in the literature (e.g., Knops, Thirion, Hubbard, Michel, \& Dehaene, 2009; Corbetta \& Shulman, 2002; Corbetta et al., 1998) and is involved in cognitive flexibility (Dajani \& Uddin, 2015) - a critical aspect of successful multistep arithmetical procedures. Another plausible suggestion made previously is that the PMC is involved in ordinality processing and sequencing of numerical elements (Lyons \& Beilock, 2013). Thus, the link between the IPS and the left PMC could reflect an integration of numerical magnitudes and an ordinality system akin to the so-called "mental number line" (Dehaene, Bossini, \& Giraux, 1993; Moyer \&
Landauer, 1967). The linearity of the mental number line, measured by asking participants to mark a certain number onto a line with two endpoints (e.g., 0-100), has been linked to mathematical abilities in childhood (Booth \& Siegler, 2008). The resting-state FC analyses also showed that the right hIP3 FC strength with the left MFG was correlated with arithmetical ability. Previous research has shown strengthened intrinsic connectivity of the IPS and the MFG after a period of repeated math tutoring sessions in children (Jolles, Supekar, et al., 2016). In addition, Ashkenazi, Black, Abrams, Hoeft, and Menon (2013) suggests that a frontoparietal network. including the IPS, PMC and MFG, may be a biomarker of MLD/DD. The relative importance of the MFG may increase with age, and a recent meta-analysis showed that adults activate the MFG to a greater degree than children during arithmetical tasks (Arsalidou, Pawliw-Levac, Sadeghi, \& Pascual-Leone, 2018). However, the exact role of the MFG in this network is not entirely clear, and the specific role of the MFG and the role of connectivity between IPS and MFG warrants further investigation. The lack of prior investigations may have been driven by a disproportionate emphasis on the IPS in number processing and dyscalculia that has diverted attention from other brain regions and networks. A recent study of adult expert mathematicians (Amalric \& Dehaene, 2016), however, found a similar frontoparietal network including the IPS and the MFG to be involved when engaged in various mathematical tasks (geometry, topology, algebra, and analysis). The authors also concluded that the MFG was the only region active at the intersection of both mathematical reasoning and more general nonmath reasoning, suggesting a role in general reflection and decision-making for MFG. Thus, the functional coupling between the IPS and the MFG may reflect a domain-general EF process, such as a working memory process.

Our resting-state FC analyses also showed a significant relationship between arithmetic ability and FC strength of the PGp and the left IFG. In terms of the overall involvement of the AG, studies showed that the AG is involved in processing of symbolic numbers and arithmetic facts (Grabner et al., 2011; Price \& Ansari, 2011; Holloway et al., 2010; Ansari, 2008). In terms of the AG and its role in arithmetic specifically, the left AG has been shown to be involved during retrieval of arithmetic facts, whereas a more distributed frontoparietal network, including the AG, was more involved while performing arithmetical procedures. Moreover, math ability has been correlated with left-lateralized AG activity, possibly reflecting a higher proficiency in processing mathematical symbols in these math-competent individuals. Our current results corroborate the importance of the AG in mathematics and more specifically the connectivity strength between the left AG and the left IFG. Previous studies have established FC patterns between the left PGp and the IFG (Uddin et al., 2010; Xiang, Fonteijn, Norris, \& Hagoort, 2010). The left AG and the left IFG are part of a language 
network that susberves phonological processing, but studies also indicate that arithmetic processing relies on these overlapping nodes (Andin et al., 2015). In a recent study, Andin et al. (2015) compared phonological processing and arithmetic processing and found overlapping substrates common to both conditions in the AG. However, upon scrutiny, the phonological condition elicited peak activations in a more anterior region of the left AG and the arithmetic condition evoked activity in the posterior portion of the left AG. The authors concluded that the PGa was more involved in processing of phonological representations and that the left PGp was proportionally more active during processing of semantic representations of long-term memory. However, Price et al. (2018) found a negative relationship of connectivity strength of the left PGp and the left SFG and arithmetic ability, but our results indicate a developmental shift in terms of involvement of the AG in mathematics insofar as adults have become more reliant on arithmetic facts during arithmetical procedures.

Concerning the role of the connectivity patterns of the SFG, which Price et al. (2018) found to be negatively related to math, our analyses indicate that the right SFG and its connection to the right hIP1 is negatively related to arithmetic ability. Price et al. (2018) argued that the negative relationship between frontoparietal hyperconnectivity and arithmetic could be due to domaingeneral network properties that impede the activity of more specialized and domain-specific networks that are important for mathematics. In our adult sample, we fail to find the same extent of the negative intrinsic FC between parietal and frontal nodes as in children (e.g., Price et al., 2018; Jolles, Ashkenazi, et al., 2016), which could be reflective of homogeneity due to cortical maturation in our adult sample.

Further investigations are warranted, however, due to some apparent discrepant and contradictory findings in the literature regarding mathematical ability and connectivity analyses in both typical and atypical development. For example, in their analyses of intrinsic FC, Price et al. (2018) found parietal interhemispheric connectivity to be predictive of arithmetic in 7- to 8-year-old children. This is inconsistent with the general notion of hyperconnectivity of the IPS in a frontoparietal network as a biomarker in MLD/DD. More specifically, Jolles, Ashkenazi, and colleagues (2016) showed that children with math difficulties exhibited greater FC between left and right IPS, as well as between IPS and other frontoparietal regions, including bilateral SPL, SFG/MFG, and SMA. The issue is further complicated by findings from another study, in which the authors found that intrinsic FC strength of the IPS with multiple cortical sites, including the pFC, increased after math tutoring (Jolles, Supekar, et al., 2016). Thus, changes in IPS connectivity were associated with arithmetic performance gains, which is in stark contrast with the notion of IPS hyperconnectivity as being indicative of abnormal math development. Trying to reconcile the aforementioned findings with our own, it seems like the relationship between IPS connectivity, both in terms of intraparietal connectivity and extraparietal connectivity in distributed frontoparietal networks, and mathematical ability may be more complex and intricate than previously thought. As such, it seems like intrinsic FC of the IPS with other cortical regions must be further investigated rather than prematurely being determined as being either inherently positive or negative for mathematical development.

\section{Specificity of Connectivity Patterns to Arithmetic}

The distributed neurocognitive network involved in performing arithmetical computations relies on cortical sites that are not uniquely involved in mathematics and basic number processing. Arithmetic calculations rely on longterm memory systems, working memory processing, and cognitive control (e.g., Fias et al., 2013). These processes are to a significant degree subserved by a central executive network that includes the IPS and the DLPFC (Sherman et al., 2014; Menon \& Uddin, 2010). Thus, it has hitherto been unclear whether there are unique subnetworks of mathematical processing or whether they are completely overlapping with neural circuits involved in $\mathrm{EF}$. To investigate the degree of neurocognitive overlap, we included three measures of $\mathrm{EF}$ as covariates in a GLM in the second analysis of resting-state FC and arithmetic ability. Previous research has shown that the dorsal attention stream comprising the SPL, motor areas, and the MFG is involved in mathematical computations (Supekar \& Menon, 2012; Menon \& Uddin, 2010; Corbetta \& Shulman, 2002). Our primary analysis discussed above revealed the very same network, with the addition of the SMG, AG, and IFG. Hence, it was unclear how much, if anything, of this network would remain significantly related to arithmetic once controlling for inhibition, shifting, and working memory. Interestingly, the analysis revealed that the involvement of the MFG, AG, and IFG was overlapping with EF to such an extent that the FC strength weakened between the ROIs and these nodes to such a degree that it did not predict arithmetic. Upon scrutiny, one might infer that the involvement of the connectivity pattern between the MFG and IPS disappeared due to the domain generality of the MFG briefly described in the previous section. The MFG is part of the DLPFC that is heavily involved in EFs and working memory ability (Barbey, Koenigs, \& Grafman, 2013). This analysis reinforces our conclusion that the role of the MFG-IPS coupling in predicting mathematics is related to EF, much as Amalric and Dehaene (2016) suggested. The coupling of the left IFG and the left PGp was related to arithmetic ability, but when controlling for $\mathrm{EF}$ it was no longer significant. The IFG is notably involved in domaingeneral cognitive control processes, such as of inhibition of irrelevant facts and motor control (Swick, Ashley, \& Turken, 2008), phonological fluency, and semantic retrieval (e.g., Katzev, Tüscher, Hennig, Weiller, \& Kaller, 
2013). Therefore, these connections are plausibly common to both $\mathrm{EF}$ processes and arithmetical calculations, and the significant coupling between the AG and the IFG and its relationship to arithmetical procedures reflect these domain-general processes.

The FC patterns that remained after controlling for EF were confined to the bilateral posterior IPS (hIP3) and the coupling to the bilateral PMC. In addition, the connectivity between the right hIP1 and the left SMG persisted. These connectivity patterns highlight the relative importance of these nodes for arithmetic relative to other domain-general processes involved in inhibition, shifting, and working memory. The link between the IPS and the PMC, as already elaborated on above, may reflect the integration of cardinal representations of numerosity and sequencing mechanisms. The consistent finding of the involvement of the PMC in task-related fMRI studies using numerical or arithmetical tasks have been concerned that the PMC activation in these paradigms may reflect motor processes, such as button presses. However, this concern was mitigated by follow-up analyses by Park et al. (2013) in their psychophysiological interaction analyses of evoked IPS connectivity. Our intrinsic connectivity analyses of resting-state data, devoid of motor interactions, corroborate the conclusion made by Park et al. (2013), thus reaffirming the relevant involvement of the IPS-PMC connectivity in arithmetical processing. The specific role of the PMC in numerical and arithmetic processing is yet to be firmly established, but the current literature allows for some tentative interpretations. For example, Lyons and Beilock (2013) showed that ordinal and cardinal judgments of symbolic numbers elicited activation patterns in the left PMC. Nevertheless, they also found that symbolic ordinality judgments activated the left PMC to greater degree and argued that the left $\mathrm{PMC}$ is involved in retrieving highly proceduralized count lists, such as the mental number line. In a task-related fMRI study in children, Park et al. (2014) established that a right parietal seed region was significantly connected to the right PMC preferentially during symbolic number processing compared with nonsymbolic comparisons. The authors suggested that this connectivity pattern subserved an integration of quantity and ordinal processing and that this connection is an index of symbolic mathematical competence in young children. We extend these findings by showing that the intrinsic FC pattern between the right IPS and the right and left PMC is indicative of arithmetical ability even in adulthood. Still, the exact mechanisms of the IPS-PMC connectivity should be investigated further to verify its role in mathematics.

The networks highlighted above also mirror some of the atypical functional activation patterns, such as the IPS, PMC, and MFG, reported in children with math difficulties (Ashkenazi, Rosenberg-Lee, Tenison, \& Menon, 2012). Our results are consistent with the notion that there may be a developmental shift in terms of reliance on the AG and the SMG, which runs somewhat contrary to Park et al. (2014), who demonstrated the level of connectivity between the right parietal cortex to the left SMG was negatively correlated with chronological age. However, one should be wary of the difference between evoked connectivity patterns driven by task demands and intrinsic connectivity patterns measured using restingstate fMRI. Future studies should disentangle different connectivity networks in combined rest and task fMRI and relate network metrics to different types of numerical stimuli, operations, and task complexity. A limitation of the current study is the absence of measures of IQ and other domain-general abilities, such as spatial ability. Inclusion of those abilities as covariates could have further reinforced the interpretations regarding the specificity of the networks and their relation to mathematics ability.

In summary, we extend previous research on children by showing a differential intrinsic connectivity pattern in adults (cf. Price et al., 2018) and that intraparietal connectivity and extraparietal connectivity of the IPS is related to arithmetical performance in a typical sample of adults. By also including measures of $\mathrm{EF}$, the analyses reveal connectivity patterns that are preferentially involved in arithmetic rather than other domain-general processes. We found overlapping connectivity patterns common to both arithmetic and EFs, insofar as that these connectivity patterns ceased to predict arithmetic when measures of EF were included as covariates. The common nodes included the MFG, AG, and IFG. In terms of unique connectivity patterns, the network comprising the IPS, PMC, and the SMG may reflect the ability to integrate symbolic and nonsymbolic magnitudes in ordered sequences that are subsequently used in arithmetical calculation procedures. Future studies should investigate how these connectivity patterns may underlie MLD/DD. It has been increasingly acknowledged that MLD/DD is a heterogeneous condition (Skagerlund \& Träff, 2016; Rubinsten \& Henik, 2009). The notion that DD is subject to subtyping introduces a further challenge of identifying underlying neurocognitive networks as candidate biomarkers, especially given the constraints present in fMRI research in terms of having adequate sample sizes for the analyzes. Still, we recommend investigations into potential neurocognitive subtyping of MLD/DD and further research into task-evoked neural connectivity patterns as well as intrinsic connectivity patterns captured using resting-state fMRI.

\section{Acknowledgments}

This research was partially supported by a grant from Linköping University (Dnr: LiU-2009-01356) awarded to Ulf Träff and partially supported by a grant awarded to Daniel Västfjäll from the Marianne and Marcus Wallenberg Foundation (Dnr: 2014-0173).

Reprint requests should be sent to Kenny Skagerlund, Department of Behavioral Sciences and Learning, Linköping University, SE-581 83 Linköping, Sweden, or via e-mail: kenny. skagerlund@liu.se. 


\section{Note}

1. Supplementary material for this paper can be retrieved from http://jedilab.weebly.com/uploads/4/9/2/3/49236491/figure_s1.tif and http://jedilab.weebly.com/uploads/4/9/2/3/4923 $\overline{6} 491 /$ table_s1.pdf.

\section{REFERENCES}

Amalric, M., \& Dehaene, S. (2016). Origins of the brain networks for advanced mathematics in expert mathematicians. Proceedings of the National Academy of Sciences, U.S.A., 113, 4909-4917.

Andin, J., Fransson, P., Rönnberg, J., \& Rudner, M. (2015). Phonology and arithmetic in the language-calculation network. Brain and Language, 143, 97-105.

Ansari, D. (2008). Effects of development and enculturation on number representation in the brain. Nature Reviews Neuroscience, 9, 278-291.

Arsalidou, M., Pawliw-Levac, M., Sadeghi, M., \& Pascual-Leone, J. (2018). Brain areas associated with numbers and calculations in children: Meta-analyses of fMRI studies. Developmental Cognitive Neuroscience, 30, 239-250.

Arsalidou, M., \& Taylor, M. J. (2011). Is $2+2=4$ ? Meta-analyses of brain areas needed for numbers and calculations. Neuroimage, 54, 2382-2393.

Ashkenazi, S., Black, J. M., Abrams, D. A., Hoeft, F., \& Menon, V. (2013). Neurobiological underpinnings of math and reading learning disabilities. Journal of Learning Disabilities, 46, 549-569.

Ashkenazi, S., Rosenberg-Lee, M., Tenison, C., \& Menon, V. (2012). Weak task-related modulation and stimulus representations during arithmetic problem solving in children with developmental dyscalculia. Developmental Cognitive Neuroscience, 2(Suppl. 1), S152-S166.

Barbey, A. K., Koenigs, M., \& Grafman, J. (2013). Dorsolateral prefrontal contributions to human working memory. Cortex, 49, 1195-1205.

Bloechle, J., Huber, S., Bahnmueller, J., Rennig, J., Willmes, K., Cavdaroglu, S., et al. (2016). Fact learning in complex arithmetic-The role of the angular gyrus revisited. Human Brain Mapping, 37, 3061-3079.

Booth, J. L., \& Siegler, R. S. (2008). Numerical magnitude representations influence arithmetic learning. Child Development, 79, 1016-1031.

Butterworth, B. (2010). Foundational numerical capacities and the origins of dyscalculia. Trends in Cognitive Sciences, 14, 534-541.

Bynner, J., \& Parsons, S. (2006). Does numeracy matter more? London: National Research and Development Centre for Adult Literacy and Numeracy, Institute of Education, University of London.

Caspers, S., Eickhoff, S. B., Geyer, S., Scheperjans, F., Mohlberg, H., Zilles, K., et al. (2008). The human inferior parietal lobule in stereotaxic space. Brain Structure and Function, 212, 481-495.

Caspers, S., Geyer, S., Schleicher, A., Mohlberg, H., Amunts, K., \& Zilles, K. (2006). The human inferior parietal cortex: Cytoarchitectonic parcellation and interindividual variability. Neuroimage, 33, 430-448.

Choi, H.-J., Zilles, K., Mohlberg, H., Schleicher, A., Fink, G. R., Armstrong, E., et al. (2006). Cytoarchitectonic identification and probabilistic mapping of two distinct areas within the anterior ventral bank of the human intraparietal sulcus. Journal of Comparative Neurology, 495, 53-69.

Corbetta, M., Akbudak, E., Conturo, T. E., Snyder, A. Z., Ollinger, J. M., Drury, H. A., et al. (1998). A common network of functional areas for attention and eye movements. Neuron, $21,761-773$.

Corbetta, M., \& Shulman, G. L. (2002). Control of goal-directed and stimulus-driven attention in the brain. Nature Reviews Neuroscience, 3, 201-215.

Dajani, D. R., \& Uddin, L. Q. (2015). Demystifying cognitive flexibility: Implications for clinical and developmental neuroscience. Trends in Neurosciences, 38, 571-578.

Dastjerdi, M., Ozker, M., Foster, B. L., Rangarajan, V., \& Parvizi, J. (2013). Numerical processing in the human parietal cortex during experimental and natural conditions. Nature Communications, 4, 2528

Dehaene, S. (2011). The number sense. New York: Oxford University Press.

Dehaene, S., Bossini, S., \& Giraux, P. (1993). The mental representation of parity and number magnitude. Journal of Experimental Psychology: General, 122, 371-396.

Dehaene, S., Spelke, E., Pinel, P., Stanescu, R., \& Tsivkin, S. (1999). Sources of mathematical thinking: Behavioral and brain-imaging evidence. Science, 284, 970-974.

De Smedt, B., \& Gilmore, C. K. (2011). Defective number module or impaired access? Numerical magnitude processing in first graders with mathematical difficulties. Journal of Experimental Child Psychology, 108, 278-292.

Feigenson, L., Dehaene, S., \& Spelke, E. (2004). Core systems of number. Trends in Cognitive Sciences, 8, 307-314.

Fias, W., Menon, V., \& Szücs, D. (2013). Multiple components of developmental dyscalculia. Trends in Neuroscience and Education, 2, 43-47.

Friston, K. J., Williams, S., Howard, R., Frackowiak, R. S. J., \& Turner, R. (1996). Movement-related effects in fMRI time-series. Magnetic Resonance in Medicine, 35, 346-355.

Gebuis, T., \& van der Smagt, M. J. (2011). False approximations of the approximate number system? PLoS One, 6, e25405.

Grabner, R. H., Ansari, D., Koshutnig, K., Reishofer, G., Ebner, F., \& Neuper, C. (2009). To retrieve or to calculate? Left angular gyrus mediates the retrieval of arithmetic facts during problem solving. Neuropsychologia, 47, 604-608

Grabner, R. H., Ansari, D., Reishofer, G., Stern, E., Ebner, F., \& Neuper, C. (2007). Individual differences in mathematical competence predict parietal brain activation during mental calculation. Neuroimage, 38, 346-356.

Grabner, R. H., Reishofer, G., Koschutnig, K., \& Ebner, F. (2011). Brain correlates of mathematical competence in processing mathematical representations. Frontiers in Human Neuroscience, 5, 130.

Harvey, B. M., Klein, B. P., Petridou, N., \& Dumoulin, S. O. (2013). Topographic representation of numerosity in the human parietal cortex. Science, 341, 1123-1126.

Holloway, I. D., Price, G. R., \& Ansari, D. (2010). Common and segregated neural pathways for the processing of symbolic and nonsymbolic numerical magnitude: An fMRI study. Neuroimage, 49, 1006-1017.

Ischebeck, A., Zamarian, L., Siedentopf, C., Koppelstätter, F., Benke, T., Felber, S., et al. (2006). How specifically do we learn? Imaging the learning of multiplication and subtraction. Neuroimage, 30, 1365-1375.

Jolles, D., Ashkenazi, S., Kochalka, J., Evans, T., Richardson, J., Rosenberg-Lee, M., et al. (2016). Parietal hyper-connectivity, aberrant brain organization, and circuit-based biomarkers in children with mathematical disabilities. Developmental Science, 19, 613-631.

Jolles, D., Supekar, K., Richardson, J., Tenison, C., Ashkenazi, S., Rosenberg-Lee, M., et al. (2016). Reconfiguration of parietal circuits with cognitive tutoring in elementary school children. Cortex, 83, 231-245.

Katzev, M., Tüscher, O., Hennig, J., Weiller, C., \& Kaller, C. P. (2013). Revisiting the functional specialization of left inferior 
frontal gyrus in phonological and semantic fluency: The crucial role of task demands and individual ability. Journal of Neuroscience, 33, 7837-7845.

Kaufmann, L., Wood, G., Rubinsten, O., \& Henik, A. (2011). Meta-analyses of developmental fMRI studies investigating typical and atypical trajectories of number processing and calculation. Developmental Neuropsychology, 36, 763-787.

Knops, A., Thirion, B., Hubbard, E. M., Michel, V., \& Dehaene, S. (2009). Recruitment of an area involved in eye movements during mental arithmetic. Science, 324, 1583-1585.

Lindskog, M., Winman, A., \& Poom, L. (2017). Individual differences in nonverbal number skills predict math anxiety. Cognition, 159, 156-162.

Lyons, I. M., \& Beilock, S. L. (2013). Ordinality and the nature of symbolic numbers. Journal of Neuroscience, 33, 17052-17061.

Mazzocco, M. M. M., Feigenson, L., \& Halberda, J. (2011). Impaired acuity of the approximate number system underlies mathematical learning disability (dyscalculia). Child Development, 82, 1224-1237.

Menon, V. (2013). Developmental pathways to functional brain networks: Emerging principles. Trends in Cognitive Sciences, 17, 627-640.

Menon, V., \& Uddin, L. Q. (2010). Saliency, switching, attention and control: A network model of insula function. Brain Structure and Function, 214, 655-667.

Moyer, R. S., \& Landauer, T. K. (1967). Time required for judgments of numerical inequality. Nature, 215, 1519-1520.

Nieder, A., \& Dehaene, S. (2009). Representation of number in the brain. Annual Review of Neuroscience, 32, 185-208.

Nieder, A., \& Miller, E. K. (2004). A parieto-frontal network for visual numerical information in the monkey. Proceedings of the National Academy of Sciences, U.S.A., 101, 7457-7462.

Park, J., Li, R., \& Brannon, E. M. (2014). Neural connectivity patterns underlying symbolic number processing indicate mathematical achievement in children. Developmental Science, 17, 187-202.

Park, J., Park, D. C., \& Polk, T. A. (2013). Parietal functional connectivity in numerical cognition. Cerebral Cortex, 23, 2127-2135.

Peters, E. (2012). Beyond comprehension: The role of numeracy in judgments and decisions. Current Directions in Psychological Science, 21, 31-35.

Piazza, M., Mechelli, A., Price, C. J., \& Butterworth, B. (2006). Exact and approximate judgements of visual and auditory numerosity: An fMRI study. Brain Research, 1106, 177-188.

Power, J. D., Barnes, K. A., Snyder, A. Z., Schlaggar, B. L., \& Petersen, S. E. (2012). Spurious but systematic correlations in functional connectivity MRI networks arise from subject motion. Neuroimage, 59, 2142-2154.

Power, J. D., Mitra, A., Laumann, T. O., Snyder, A. Z., Schlaggar, B. L., \& Petersen, S. E. (2014). Methods to detect, characterize, and remove motion artifact in resting state fMRI. Neuroimage, $84,320-341$.
Price, G. R., \& Ansari, D. (2011). Symbol processing in the left angular gyrus: Evidence from passive perception of digits. Neuroimage, 57, 1205-1211.

Price, G. R., Yeo, D. J., Wilkey, E. D., \& Cutting, L. E. (2018). Prospective relations between resting-state connectivity of parietal subdivisions and arithmetic competence. Developmental Cognitive Neuroscience, 30, 280-290.

Rosenberg-Lee, M., Ashkenazi, S., Chen, T., Young, C. B., Geary, D. C., \& Menon, V. (2015). Brain hyper-connectivity and operation-specific deficits during arithmetic problem solving in children with developmental dyscalculia. Developmental Science, 18, 351-372.

Rubinsten, O., \& Henik, A. (2009). Developmental dyscalculia: Heterogeneity might not mean different mechanisms. Trends in Cognitive Sciences, 13, 92-99.

Sherman, L. E., Rudie, J. D., Pfeifer, J. H., Masten, C. L., McNealy, K., \& Dapretto, M. (2014). Development of the default mode and central executive networks across early adolescence: A longitudinal study. Developmental Cognitive Neuroscience, 10, 148-159.

Skagerlund, K., \& Träff, U. (2016). Number processing and heterogeneity of developmental dyscalculia: Subtypes with different cognitive profiles and deficits. Journal of Learning Disabilities, 49, 36-50.

Supekar, K., \& Menon, V. (2012). Developmental maturation of dynamic causal control signals in higher-order cognition: A neurocognitive network model. PLoS Computational Biology, 8, e1002374.

Swick, D., Ashley, V., \& Turken, A. U. (2008). Left inferior frontal gyrus is critical for response inhibition. BMC Neuroscience, 9, 102.

Uddin, L. Q., Supekar, K., Amin, H., Rykhlevskaia, E., Nguyen, D. A., Greicius, M. D., et al. (2010). Dissociable connectivity within human angular gyrus and intraparietal sulcus: Evidence from functional and structural connectivity. Cerebral Cortex, 20, 2636-2646.

van der Sluis, S., de Jong, P. F., \& van der Leij, A. (2004). Inhibition and shifting in children with learning deficits in arithmetic and reading. Journal of Experimental Child Psychology, 87, 239-266.

Wechsler, D. (2008). Wechsler Adult Intelligence Scale (4th ed.). San Antonio, TX: Pearson Assessment.

Wu, S. S., Chang, T. T., Majid, A., Caspers, S., Eickhoff, S. B., \& Menon, V. (2009). Functional heterogeneity of inferior parietal cortex during mathematical cognition assessed with cytoarchitectonic probability maps. Cerebral Cortex, 19, 2930-2945.

Xiang, H. D., Fonteijn, H. M., Norris, D. G., \& Hagoort, P. (2010). Topographical functional connectivity pattern in the perisylvian language networks. Cerebral Cortex, 20, 549-560.

Xu, F., \& Spelke, E. S. (2000). Large number discrimination in 6-month-old infants. Cognition, 74, B1-B11.

Yan, C.-G., Wang, X.-D., Zuo, X.-N., \& Zang, Y.-F. (2016). DPABI: Data processing $\&$ analysis for (resting-state) brain imaging. Neuroinformatics, 14, 339-351. 